Journal of Patient-Centered coAdvocateAuroraHealth

Research and Reviews

Volume 5

Issue 1 -- Health Disparities and Inequities: Part

Article 2

II

$1-30-2018$

\title{
In-Home Delivery of Constraint-Induced Movement Therapy via Virtual Reality Gaming
}

\author{
Alexandra L. Borstad \\ Roger Crawfis \\ Kala Phillips \\ Linda Pax Lowes \\ David Maung \\ Ryan McPherson \\ Amelia Siles \\ Lise Worthen-Chaudhari \\ Lynne V. Gauthier
}

Follow this and additional works at: https://aah.org/jpcrr

Part of the Equipment and Supplies Commons, Movement and Mind-Body Therapies Commons, Musculoskeletal, Neural, and Ocular Physiology Commons, Musculoskeletal System Commons, Orthopedics Commons, and the Other Rehabilitation and Therapy Commons

\section{Recommended Citation}

Borstad AL, Crawfis R, Phillips K, Pax Lowes L, Maung D, McPherson R, Siles A, Worthen-Chaudhari L, Gauthier LV. In-home delivery of constraint-induced movement therapy via virtual reality gaming. J Patient Cent Res Rev. 2018;5:6-17. doi: 10.17294/2330-0698.1550

Published quarterly by Midwest-based health system Advocate Aurora Health and indexed in PubMed Central, the Journal of Patient-Centered Research and Reviews (JPCRR) is an open access, peer-reviewed medical journal focused on disseminating scholarly works devoted to improving patient-centered care practices, health outcomes, and the patient experience. 


\title{
In-Home Delivery of Constraint-Induced Movement Therapy via Virtual Reality Gaming
}

\author{
Alexandra L. Borstad, PhD, PT, ${ }^{1}$ Roger Crawfis, PhD, ${ }^{2}$ Kala Phillips, MS,${ }^{3}$ Linda Pax Lowes, PhD, ${ }^{4}$ \\ David Maung, PhD, ${ }^{5}$ Ryan McPherson, PhD, ${ }^{6}$ Amelia Siles, DPT, ${ }^{7}$ Lise Worthen-Chaudhari, MFA, MS, ${ }^{8}$ \\ Lynne V. Gauthier, $\mathrm{PhD}^{8}$ \\ ${ }^{1}$ Department of Physical Therapy, College of St. Scholastica, Duluth, MN; ${ }^{2}$ Department of Biomedical Engineering, The \\ Ohio State University, Columbus, $\mathrm{OH} ;{ }^{3}$ Department of Psychological and Brain Sciences, University of Louisville, $\mathrm{KY}$; \\ ${ }^{4}$ Nationwide Children's Hospital, Columbus, $\mathrm{OH} ;{ }^{5} \mathrm{Battelle}$, Columbus, $\mathrm{OH} ;{ }^{6}$ Department of Electrical and Computer \\ Engineering, The Ohio State University, Columbus, $\mathrm{OH}$; ${ }^{7}$ Division of Physical Therapy, The Ohio State University, \\ Columbus, $\mathrm{OH} ;{ }^{8}$ Department of Physical Medicine and Rehabilitation, The Ohio State University, Columbus, $\mathrm{OH}$
}

Purpose People with chronic hemiparesis are frequently dissatisfied with the recovery of their hand and arm, yet many lack access to effective treatments. Constraint-induced movement therapy $(\mathrm{Cl}$ therapy) effectively increases arm function and spontaneous use in persons with chronic hemiparesis. The purpose of this study was to determine the feasibility and measure safety and outcomes of an in-home model of delivering $\mathrm{Cl}$ therapy using a custom, avatar-based virtual reality game.

Methods Seventeen individuals with chronic hemiparesis participated in this pretest/posttest quasi-experimental design study. The 10-day intervention had three components: 1) high-repetition motor practice using virtual reality gaming; 2) constraint of the stronger arm via a padded restraint mitt; and 3) a transfer package to reinforce arm use. Feasibility of the intervention was evaluated through comparison to traditional Cl therapy and through participants' subjective responses. The primary outcome measures were the Wolf Motor Function Test (WMFT) and the Motor Activity Log quality of movement scale (MAL-QOM).

Results On average, participants completed $17.2 \pm 8$ hours and 19,436 repetitions of motor practice. No adverse events were reported. Of 7 feasibility criteria, 4 were met. WMFT rate and MAL-QOM increased, with effect size (Cohen's $d$ ) of 1.5 and 1.1, respectively.

Conclusions This model of delivering $\mathrm{Cl}$ therapy using a custom, avatar-based virtual reality game was feasible, well received, and showed preliminary evidence of being a safe intervention to use in the home for persons with chronic hemiparesis. (J Patient Cent Res Rev. 2018;5:6-17.)

Keywords stroke; upper extremity; virtual reality exposure therapy; feasibility

$\mathrm{I}$ mpairments in the hand and arm result in diminished quality of life for persons poststroke, ${ }^{1}$ with only $6 \%$ of chronic stroke survivors feeling satisfied with the recovery of their impaired hand and arm. ${ }^{2}$ Constraint-induced movement therapy (CI therapy) is an intensive method of motor rehabilitation in which the participant is encouraged to use the more affected

Correspondence: Lynne V. Gauthier, PhD, The Ohio State University, 480 Medical Center Drive, Columbus, OH, 43210, T: +1-614-293-3830,

Email: lynne.gauthier@osumc.edu arm for all daily activities while reducing use of the less affected arm during the treatment period. The goal is to improve arm function and overcome the learned behavior of non-use. ${ }^{3}$

CI therapy substantially increases arm function and spontaneous use of the more affected $\operatorname{arm}^{3-5}$ and promotes structural ${ }^{6}$ and functional ${ }^{7}$ brain plasticity. Despite a high level of evidence for effectiveness for improving arm function and use, ${ }^{8-10} \mathrm{CI}$ therapy remains inaccessible to most patients due to limited availability of treatment programs, limited insurance coverage, 
and high cost. ${ }^{11}$ Broader challenges for persons poststroke, such as residing remotely from a treatment center or having limited access to transportation, likely contribute to the disparity in access to evidence-based rehabilitation and to overall dissatisfaction with upper limb recovery. ${ }^{11,12}$

A potential solution to the challenges of access to CI therapy and the need for ongoing rehabilitation is increasingly affordable gaming technology. Researchers have begun to pilot in-home, patient-led virtual reality gaming for upper limb rehabilitation in individuals with cerebral palsy ${ }^{13,14}$ and stroke. ${ }^{15-20}$ Clinically meaningful improvements in motor function were reported in 4 of 5 small-sample prospective studies. ${ }^{15,17-19}$ However, minimal gains in everyday use of the weaker arm (an outcome of importance to stakeholders) were realized. ${ }^{17,18}$ The intensity of training in these early studies also was relatively low, with participants engaged in game play from 17 to 37 minutes per study day over 4 to 12 weeks (number of repetitions of task practice were not reported). Thus, it remained to be determined whether stroke survivors would adhere to unsupervised high-repetition practice through in-home gaming at training intensities characteristic of CI therapy ( 3 hours per day). ${ }^{21}$ It also was unknown whether the "transfer package" of CI therapy ${ }^{22}$ that promotes carryover of motor training to daily activities could be effective given significantly reduced therapist contact.

The purposes of this study were threefold: 1) to determine the feasibility and monitor safety of inhome delivery of CI therapy via virtual reality gaming; 2) to determine the intensity of practice (number of repetitions per time) that can be achieved through continuous therapeutic game play; and 3) to document change in motor performance for the purpose of planning larger controlled studies. ${ }^{23}$ Herein, we report an account of intense, in-home, minimally supervised, high-repetition motor practice via virtual reality gaming that is based on a well-established rehabilitation program for treating upper limb hemiparesis.

\section{METHODS}

\section{Design, Setting, Participants}

This prospective cohort study used a pretest/posttest quasi-experimental design and was conducted in an academic medical center. A total of 17 community- dwelling individuals with upper extremity hemiparesis, who resided within 50 miles of the academic medical center, were enrolled. Participants provided written informed consent. Demographic information about participants is provided in Table 1 .

Table 1. Baseline Participant Characteristics ( $N=17)$

\begin{tabular}{lc}
\hline Measure & Pretest mean (SD) \\
\hline Age, years & $49(19.8)$, range 14-69 \\
Sex & 10 male \\
Chronicity, months & $37(19)$ \\
Right side affected & 9 \\
Cause of hemiparesis & 16 stroke, 1 tumor resection \\
Affected side was dominant & 8 \\
MoCA score & $22(5)$ \\
PHQ-9 severity grade & $5(5)$ \\
Brief Kinesthesia Test, cm & $8.7(5.2)$ \\
Impaired touch-test score & 14 \\
Resided urban/suburban/rural & $4 / 6 / 7$ \\
\hline
\end{tabular}

MoCA, Montreal Cognitive Assessment; PHQ-9, Patient Health Questionnaire-9; SD, standard deviation.

The Ohio State University biomedical institutional review board approved the study. The recruiting process took place from September 2012 to December 2014, and participants were recruited through advertisement, mailings, local stroke support groups and care providers. Laboratory research assistants screened potential participants for eligibility. Of 17 participants, 16 completed the intervention; one was withdrawn following an unrelated medical event that rendered her unable to complete the intervention within the maximally allotted 3-week period. This trial is registered at ClinicalTrials.gov (NCT03005457).

\section{Criteria}

Potential participants met the following study criteria, which were similar to those used in the EXCITE trial of CI therapy ${ }^{24}$ : 1) hemiparesis for $\geq 6$ months; 2) capacity to provide informed consent; 3 ) not receiving concurrent outpatient rehabilitation for their upper extremity; 4) no history of having received CI therapy or modified CI therapy; 5) $30^{\circ}$ active shoulder flexion, $20^{\circ}$ active elbow extension, at least $10^{\circ}$ active wrist extension, at least $10^{\circ}$ thumb abduction/extension, and at least $10^{\circ}$ extension in at least 2 digits; 6) the expressed willingness to wear a restraint mitt on the less affected upper extremity for the majority of waking 
hours; and 7) not having received Botox injections within 12 weeks of study participation.

\section{Intervention}

In collaboration with therapists and stroke survivors, an in-home therapist-as-consultant patient-centered model of CI therapy was designed to leverage the advantages of both new gaming technology and highly trained practitioners. The therapist's role was to instruct the participant in the desired movement mechanics for game play, customize the game, and to promote carryover of training (increased use of the weaker arm during daily activities). The role of the gaming system was to reduce access barriers and render motor practice more engaging by promoting high-intensity in-home motor practice with the desired movement mechanics.

\section{Game Design}

A flexible video game software platform, called Recovery Rapids (Games That Move You PBC, Columbus, $\mathrm{OH}),{ }^{25-27}$ was designed to promote both personalized game play and motor practice that maximally challenged participants (Figure 1). It captured movements of the participants' more affected upper extremity via the Xbox 360 Kinect sensor (Microsoft Corporation, Redmond, WA) and an openpalmed glove equipped with axial accelerometers and flex sensors (detecting wrist supination/pronation and thumb, index, and middle finger flexion/extension) to drive game play. While the therapist prompted the participant to perform his/her best possible movement attempts, the software stored the current ability level of the participant for each movement. When the therapist was no longer present, the game would only respond to movements at or above the participant's current ability level. As a participant gained mastery, Recovery Rapids required successively larger movements to trigger a game action. ${ }^{25}$ For example, to move the kayak forward in the game, the participant completed a sagittal plane shoulder flexion toward extension movement. As a participant improved, the game required successively greater shoulder flexion and/or elbow extension. ${ }^{26}$

The therapist customized the rehabilitation program to each individual by specifying the relative frequency of different game actions that are each associated with a therapeutic movement (Table 2). Details regarding the algorithm used to achieve therapy progression and personalization have been reported by Maung et al. ${ }^{26}$

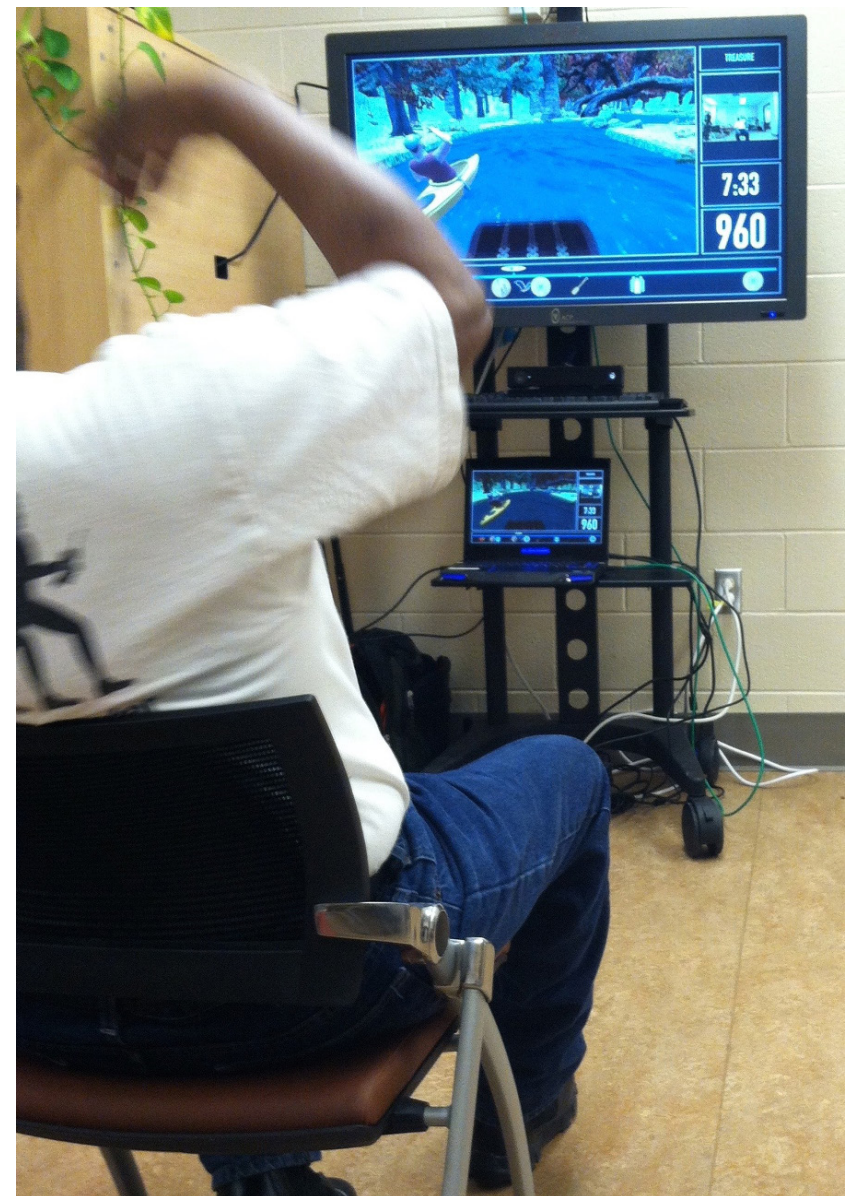

Figure 1. In Recovery Rapids, movements of the participants' more affected upper extremity, captured via the Xbox 360 Kinect sensor, drive game play. The participant's posture and reaching action are reflected in the avatar's position.

Table 2 details game gestures, the motions required to successfully complete the gesture, and how that motion is progressed. Consistent with the intensity of CI therapy, ${ }^{21}$ participants were asked to play Recovery Rapids 3 hours per day for 10 study days over 2 weeks without therapist supervision.

Daily monitoring of the use of the more affected arm via the Motor Activity Log (MAL), a portion of the transfer package of CI therapy, was embedded within game play. A series of 3 MAL questions with problemsolving modules were distributed approximately every 20 minutes throughout game play (Figure 2).

\section{Therapist Home Consultation Visits}

Five total hours of therapist consultation was conducted with each participant. The initial visit ( 2 hours) included review of the study protocol, instruction 
Table 2. Description of Gestures

\begin{tabular}{|c|c|c|}
\hline Game action & Motion required & How progressed \\
\hline $\begin{array}{l}\text { Row to make kayak travel } \\
\text { down-river }\end{array}$ & $\begin{array}{l}\text { Sagittal plane shoulder flexion/extension with } \\
\text { elbow extension }\end{array}$ & $\begin{array}{l}\text { Increase shoulder flexion } \\
\text { Increase elbow extension }\end{array}$ \\
\hline $\begin{array}{l}\text { Steer boat toward the } \\
\text { hemiparetic side }\end{array}$ & Shoulder abduction with elbow extension & $\begin{array}{l}\text { Increase shoulder abduction } \\
\text { Increase elbow extension }\end{array}$ \\
\hline $\begin{array}{l}\text { Steer boat toward the less } \\
\text { affected side }\end{array}$ & Horizontal shoulder adduction across midline & $\begin{array}{l}\text { Increase shoulder adduction } \\
\text { Increase elbow extension }\end{array}$ \\
\hline Catch fish with a net & Elbow flexion/extension & $\begin{array}{l}\text { Increase elbow flexion } \\
\text { Increase elbow extension }\end{array}$ \\
\hline Collect bottles from a river & Elbow flexion/extension and grasp/release & $\begin{array}{l}\text { Increase elbow flexion } \\
\text { Increase elbow extension } \\
\text { Increase thumb and finger extension }\end{array}$ \\
\hline $\begin{array}{l}\text { Catch parachute to receive } \\
\text { supplies }\end{array}$ & $\begin{array}{l}\text { Forearm supination with shoulder flexion and } \\
\text { elbow extension }\end{array}$ & $\begin{array}{l}\text { Increase shoulder flexion } \\
\text { Increase forearm supination } \\
\text { Increase elbow extension } \\
\text { Increase duration of held posture }\end{array}$ \\
\hline Picking fruits from bushes & $\begin{array}{l}\text { Finger flexion/extension and thumb abduction/ } \\
\text { adduction with shoulder flexion to position hand } \\
\text { over target }\end{array}$ & Increase thumb and finger extension \\
\hline Turn over card & $\begin{array}{l}\text { Forearm supination with shoulder flexion to } \\
\text { position hand over target }\end{array}$ & Not progressed \\
\hline Flick letters in a word puzzle & $\begin{array}{l}\text { Wrist extension with shoulder flexion to position } \\
\text { hand over target }\end{array}$ & Not progressed \\
\hline
\end{tabular}

in Recovery Rapids game play, customization of Recovery Rapids to the individual, and implementation of the CI therapy "transfer package." 22 The second visit consisted of establishing a treatment contract in which the participant outlined daily tasks to be performed exclusively with the weaker arm, obtaining participant "buy-in" to wear a padded mitt restraint on the stronger arm for 10 hours daily, and collaboratively constructing an additional home-practice program for 30 minutes per day that consisted of goal-related functional tasks to be completed independently by the participant. Goal-related functional tasks were unique to each participant. They shared the characteristics of being salient to the participant, having potential for improvement, and being safe for the participant to perform without supervision. Subsequent 1-hour visits included discussing compliance with mitt use, reviewing goal-related functional tasks, completing guided problem-solving related to arm use for daily activities, troubleshooting game play, and adjusting Recovery Rapids as needed to optimally challenge the participant.

\section{Measurements}

Demographics: Demographic and outcome measures were collected in an academic medical center research laboratory. Baseline gross cognitive and emotional functioning were assessed via the Montreal Cognitive Assessment (MoCA) and the Patient Health Questionaire-9 (PHQ-9), ${ }^{28}$ respectively. The MoCA is a brief measure of global cognitive function for which scores less than 25 evidence impairment. ${ }^{29}$ The PHQ-9 is a 9-item questionnaire that suggests presence of depression and can be used to grade severity $(\leq 4=$ no depression, 5 to $9=$ mild, 10 to $14=$ moderate, 15 to $19=$ moderately severe, and 20 to $27=$ severe depression)..$^{30}$

Feasibility: Seven criteria, drawn primarily from traditional CI therapy, were used to determine feasibility of the intervention: 1) adherence to total hours training time of CI therapy (30 hours of play in 10 days $^{21}$ ); 2) adherence to active movement time of CI therapy estimated to be 6.25 hours in 10 days (Edward Taub, personal communication, January 25, 2016); 3) mitt use 


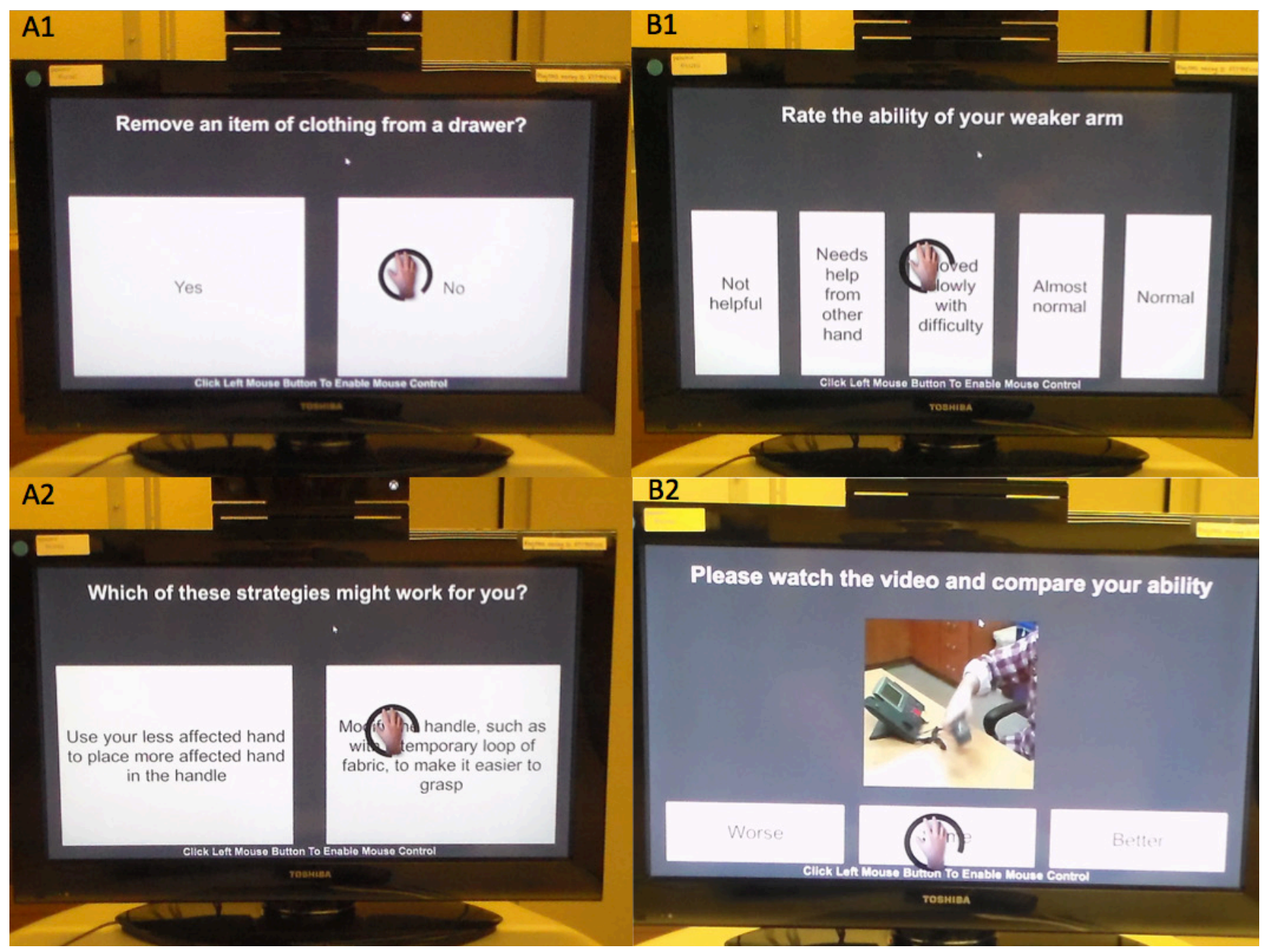

Figure 2. To complete the in-game Motor Activity Log (MAL), participants view questions and videos and select responses by hovering a cursor (controlled by the affected upper extremity) over the desired response. Panel A1: Participant indicates if they have removed an item of clothing from a drawer in the last 2 days. Panel A2: When participants indicate 'No,' they are prompted to select a strategy that might work for them next time they try the task. Panel B1: When participants select 'Yes' (that they have completed the task in the last 2 days), they are asked to rate their ability, in this case using the phone. Panel B2: To anchor the responses, they are then shown a video example of a person performing that task with that quality of movement (QOM) rating and have the opportunity to adjust their rating up or down. Based on their final rating, the software assigns the appropriate MAL-QOM score to their response.

(100 hours in 10 days $\left.^{21}\right)$; 4) participation in therapist visits; 5) completion of in-game administration of the MAL (6 total administrations $\left.{ }^{21}\right) ; 6$ ) participant subjective response regarding feasibility; and 7) participant subjective response regarding usefulness.

Safety: A unique safety issue anticipated because of the high-repetition motor practice in this protocol was shoulder pain. The analytic approach used to evaluate safety of the intervention was counts of serious studyrelated adverse events, assessed via participant selfreport upon inquiry by the consulting therapist at each visit. A serious study-related adverse event was defined as any untoward medical event that could be considered to have occurred due to study participation and either required medical attention or precluded participants from continuing the study. The criterion for safety, established a priori, was zero adverse events during the intervention.

\section{Outcomes}

Outcome measures were collected within 3 weekdays prior to and following the completion of training, with three exceptions that resulted from participants rescheduling testing or training (pretest 11 days prior to beginning the intervention for 1 participant, posttests 6 
and 14 days following completion of the intervention for 2 participants). Independent testers with no intellectual or financial stake in Recovery Rapids, and who had limited knowledge of the treatment protocol and who did not participate in treatment, completed the testing. Testers were not informed of pre- or posttreatment condition, but "unblinding" may have occurred in some cases (eg, if a tester remembered a participant from a prior testing session).

In keeping with the fundamental principles of patientcentered research, we selected outcomes of importance to people with chronic hemiparesis. During the study design process, we surveyed 10 individuals with chronic stroke who participated in a study-specific patient advisory group ${ }^{31}$ and recorded their common frustrations with their upper extremity function. We then selected outcome measures that captured each of these aspects of performance. Table 3 includes their comments and the outcome measures chosen.

Table 3. Outcomes of Importance to Study Participants

\begin{tabular}{lc}
\hline Comment & $\begin{array}{c}\text { Outcome } \\
\text { measure }\end{array}$ \\
\hline "I tend to drop things" & $\begin{array}{c}\text { touch-test } \\
\text { "I can't reach above my shoulder" }\end{array}$ \\
WMFT, ARAT \\
"I can't feel it so I don't trust it" & touch-test \\
"I forget I have something in my hand" & touch-test \\
"I forget to use my hand" & Motor Activity Log \\
"It takes too long [to use my weaker hand]" & WMFT \\
"...not knowing what my arm is doing & $\begin{array}{c}\text { Brief } \\
\text { unless I am looking at it," "It's so } \\
\text { effortful [to use my vision]" }\end{array}$ \\
\hline
\end{tabular}

ARAT, Action Research Arm Test; WMFT, Wolf Motor Function Test.

Primary Outcomes: The Wolf Motor Function Test $(\mathrm{WMFT})^{32}$ and $\mathrm{MAL}^{3}$ were chosen as the primary outcome measures for this study because they have excellent psychometric properties, measure distinct aspects of motor ability that are important to the post-stroke community, and were utilized as primary outcome measures in the seminal papers on CI therapy. ${ }^{3-5,24,33}$ The rate metric was used for scoring the WMFT because it enhances interpretability and is known to be more sensitive in participants with moderate motor deficit than scoring by performance time. ${ }^{32}$ The WMFT rate metric was favored over other metrics of motor ability (eg, Action Research Arm Test [ARAT], WMFT-Functional Ability Scale) because advisory board participants reported being more troubled by slow task performance than by their movements appearing less "normal."

Exploratory Outcomes: The ARAT, ${ }^{34-36}$ a 19-item measure of quality of movement (QOM) during grasp, grip, pinch, and gross movement, served as an exploratory motor measure. Measures associated with somatosensory functioning of importance to stakeholders (Table 2) are categorical measures, or are less sensitive to change following rehabilitation, and were thus considered exploratory. The Brief Kinesthesia Test quantifies error in targeted reaching to evaluate kinesthetic impairment. ${ }^{37,38}$ It can detect differences in kinesthetic performance between people with mildmoderate hemiparesis post-stroke and age-matched controls. ${ }^{39}$ A cut point of $>6.5 \mathrm{~cm}$ error was used to indicate impairment in kinesthetic sense. A Touch-Test ${ }^{\mathrm{TM}}$ monofilament esthesiometer quantifies the threshold of index finger touch perception in grams ${ }^{40}$ with acceptable interrater reliability (intraclass correlation coefficient [ICC]: $0.77-0.99)^{41}$ and test-retest reliability (ICC: $0.69-0.71){ }^{42}$ Touch-test data were log transformed as recommended for normalization. ${ }^{43}$ The dominant hand was identified through self-report.

Participant active play-time and the number of gesture repetitions completed were calculated by analyzing data files that logged each gesture detected and their time stamps. Epochs in which participants stepped away from the game (defined as 5 minutes without a gesture recorded) were excluded from the playtime calculation. To reduce the possibility of double counting gestures due to cross talk (simultaneous triggering of two gestures), the parsing code counted no more than 1 gesture per second. Gesture counts were cross-validated by visually counting the gestures performed by a novice player with hemiparesis in the laboratory during 30 minutes of play. The parsing code was $9 \%$ more conservative than the visual count during this validation, thus it provided a conservative estimate of gestures completed. Participant active play-time and the number of gestures completed per hour were strongly correlated $(\mathrm{r}=0.92, \mathrm{P}<0.0001)$. Mean values are found in Table 4. 


\section{Data Analysis}

Data were examined for normality using ShapiroWilk test $(\mathrm{P} \geq 0.05)$. Student's paired t-tests were run separately for primary (WMFT rate, MAL-QOM) and exploratory outcome variables to estimate the treatment effect. For the primary outcome analysis, alpha was set at $\alpha$ of 0.025 after Bonferroni correction for two comparisons. Analysis of exploratory outcome measures was conducted without correction for multiple comparisons to identify trends in the data, defined as $\mathrm{P} \leq 0.1$, which may form the foundation for future work. Effect size was calculated for outcomes that were statistically different at posttest using a standardized measure of effect Cohen's $d .{ }^{44}$

Justified by the small number of participants who did not complete the study ( 1 of 17), only complete data sets were analyzed. ${ }^{45}$ There were no missing data for the primary outcomes; $2 \%$ of the data was missing overall. No systematic differences between missing and nonmissing data were detected, thus data were considered to be missing at random. Restricted maximum likelihood was chosen as the method to impute missing data because it is recommended when missing data are random in relatively small data sets such as this. ${ }^{46}$ Study data were managed using REDCap as hosted at The Ohio State University. Statistics were performed using JMP ${ }^{\circledR}$ Pro 11.0.0 (SAS Institute Inc., Cary, NC) and built-in functions in Microsoft ${ }^{\circledR}$ Excel $^{\circledR}$ for Mac 2011 (Microsoft Corporation).

\section{RESULTS \\ Feasibility}

Of the 7 feasibility criteria, 4 were met. These included achieving the amount of active movement time of CI therapy, $100 \%$ participation in home visits, and participant subjective responses regarding the feasibility and usefulness of the intervention. Feasibility measures, criteria, and outcomes are given in Table 4.

\section{Safety}

No serious study-related adverse events were reported during this study, thus it met the a priori criteria for safety. One left-affected participant experienced the onset of shoulder pain when attempting to move the

Table 4. Feasibility Measures, Criteria, and Outcomes

\begin{tabular}{|c|c|c|c|}
\hline Feasibility measure & Criteria & Mean outcome & Met / Not met \\
\hline Total training time, hours & 30 & $17.2 \pm 8(58 \%)$ & Not met \\
\hline Active movement time, hours & 6.25 & $17.2 \pm 8(275 \%)$ & Met \\
\hline Mitt use, self-reported hours & 100 & $48 \pm 34(48 \%)$ & Not met \\
\hline In-game MAL completions & 6 & $3.2(53 \%)$ & Not met \\
\hline Participation in home visits & $100 \%$ & $100 \%$ & Met \\
\hline Participant subjective: feasibility & $\geq 4$ & & Met \\
\hline $\begin{array}{l}\text { Using the game is [ } 1=\text { harder, } 4=\text { same, } 7=\text { easier] than } \\
\text { other rehabilitation I have done for my arm. }\end{array}$ & & 4.7 & \\
\hline $\begin{array}{l}\text { I felt [ } 1=\text { uncomfortable, } 4=\text { same, } 7=\text { comfortable] playing } \\
\text { the game in my home. }\end{array}$ & & 6.6 & \\
\hline Participant subjective: usefulness & $\geq 4$ & & Met \\
\hline $\begin{array}{l}\text { How useful was the game to your rehabilitation? } \\
\text { [1=not at all, } 4=\text { =neutral, } 7=\text { extremely useful] }\end{array}$ & & 6.3 & \\
\hline $\begin{array}{l}\text { The game is }[1=\text { less, } 4=\text { same, } 7=\text { more }] \text { effective } \\
\text { than other rehabilitation I have done for my arm. }\end{array}$ & & 5.9 & \\
\hline
\end{tabular}

MAL, Motor Activity Log. 
Table 5. Outcome Measures

\begin{tabular}{lcccc}
\hline Feasibility measure & Pretest mean (SD) & Posttest mean (SD) & $\boldsymbol{P}$ & Effect size \\
\hline Primary outcome & $\mathrm{n}=16$ & $\mathrm{n}=16$ & & \\
WMFT rate/60 seconds & $22.4(9.3)$ & $28.1(11.4)$ & $<0.001$ & 1.5 \\
MAL-QOM, 0-5 scale & $1.5(1.1)$ & $2.2(1.3)$ & $<0.001$ & 1.1 \\
Exploratory outcome & $\mathrm{n}=16$ & $\mathrm{n}=16$ & & \\
$\quad$ ARAT score & $33.9(17.9)$ & $35.1(19.1)$ & 0.33 & $\mathrm{NA}$ \\
Touch-test log, g & $0.03(1.40)$ & $-0.31(0.88)$ & 0.08 & $\mathrm{NA}$ \\
Brief Kinesthesia Test, cm & $8.7(5.2)$ & $8.2(5.4)$ & 0.60 & $\mathrm{NA}$ \\
\hline
\end{tabular}

ARAT, Action Research Arm Test; MAL-QOM, Motor Activity Log for quality of movement; SD, standard deviation; WMFT, Wolf Motor Function Test.

kayak toward the hemiparetic side using shoulder abduction on day 3 of game play. During consultation, the therapist decreased the required range of shoulder abduction to trigger the game action and the participant completed the study, playing 33 hours total, without further symptoms of shoulder pain.

\section{Intensity of Practice}

Participants completed $1130 \pm 321$ repetitions of upper limb movements per hour of game play. The participant who played at the slowest pace far exceeded the recently characterized high dose for upper limb training of 300 repetitions per hour. ${ }^{47}$

\section{Outcomes}

Primary and exploratory outcomes are shown in Table 5. The mean (standard deviation), median, and interquartile range for within-subjects change on the WMFT (rate/60 seconds) and MAL-QOM (0-5 scale) were 5.8 (3.7), 5.8, 2.7-9.4 and 0.74 (0.66), 0.46, $0.28-1.11$, respectively. Gaming CI therapy showed a large effect on the primary outcome measures of WMFT rate and MAL-QOM. ${ }^{44}$ Average MAL-QOM change of 0.7 on the 5-point scale exceeded the proposed minimal clinically important change score of $10 \%$ of the total range. ${ }^{48}$ The 4 participants with the most severe touch impairment (monofilament threshold of $\geq 300 \mathrm{~g}$ ) at pretest had improved tactile sensation by one category at posttest. Overall touch-test scores were not statistically different $(\mathrm{P}=0.08)$.

\section{DISCUSSION}

This pilot study demonstrated that despite unsupervised high-repetition motor practice, the intervention was feasible overall and safe within this trial. Consistent with a recent gaming study by Combs and colleagues, ${ }^{49}$ these findings demonstrate that persons with chronic hemiparesis tolerate - and choose to engage in high-repetition motor practice when provided with a motivating environment. High-repetition motor practice has been associated with neural plasticity and may improve skill performance..$^{50}$

The high target of 3 hours of active game play per day chosen for this study, based on treatment-time dosing of traditional in-clinic CI therapy, ${ }^{21,24}$ was not achieved by most participants. Upper extremity activity is nearly continuous and, at times, fast-paced while playing Recovery Rapids, whereas traditional CI therapy includes frequent breaks. It is possible that some participants experienced fatigue while following the protocol. Although 30 hours of game play over 10 treatment days overall was not feasible, the amount of active motor practice participants achieved far exceeded 30 hours of in-clinic CI therapy (17.2 vs 6.25 hours). ${ }^{21}$ Participants in this trial thus received doses of motor practice that are likely comparable or greater than the doses most frequently utilized among CI therapy studies demonstrating clinically meaningful improvement. ${ }^{9}$

Overall, compliance with in-game MAL problemsolving and mitt use was poor. Qualitative feedback from participants suggested two main themes to explain these findings. First, the salience of the game overshadowed the importance of the transfer package (including in-game MAL), despite the emphasis placed on it by the therapist during consultation visits. 
Second, substantially reduced therapist contact time made participants feel less accountable for performing therapeutic tasks that are objectionable (mitt restraint) or less engaging (in-game MAL). The mitt was particularly disliked by most participants because it was time-consuming to get on and off, impeded hygiene (eg, washing hands), and got dirty. The result was that participants received a much lower "dose" of the transfer package techniques known to be important for carryover of motor gains to daily activities. ${ }^{21}$ Omitting the behavioral aspects of the transfer package (eg, MAL, problem-solving) is known to substantially reduce improvements in everyday arm use that typically accompany CI therapy ${ }^{21}$ whereas omitting the mitt restraint has historically had minimal adverse effect. $^{51}$

In summary, all feasibility metrics that support the potential for positive clinical outcomes have been met, with the exception of a lower dose of the MAL with problem-solving. Based on these findings, our stroke community partners have suggested that future work omit the mitt restraint and emphasize the importance of the transfer package in at least two ways - an educational video that emphasizes the importance of the transfer package and more salient prompting from the therapist (ie, reviewing the MAL at the beginning of each session and engaging in more face-to-face active problem-solving)..$^{52,53}$

Consistent with poorer adherence to the selfassessment and problem-solving components of the transfer package (ie, MAL), improvement in quality of upper extremity arm use for daily activities was less than half as large as that observed with traditional CI therapy, ${ }^{21}$ though still clinically meaningful. Additional factors also may have reduced carryover. For example, participants in traditional CI therapy protocols who interacted with therapists for a total of 35 hours (30 hours of motor practice plus 5 hours of transfer package) had substantially more opportunity to engage in actively problem-solving arm use for daily activities during short breaks within the 3-hour daily sessions of motor training than did the participants in this trial whose interaction with the therapist was limited to the 5 hours of consultation focused explicitly on the transfer package. This study sample was more inclusive than prior CI therapy studies, with a high incidence of cognitive/memory impairment among participants, which may interfere with carryover of strategies for improved arm use. It is also possible that game gestures do not approximate real-life demands, such as grasping an object, as well as traditional inclinic CI therapy tasks do, and that this limits carryover to activities of daily living.

Performance speed (WMFT rate metric) improved significantly following the intervention, and this improvement was nearly identical to that found in prior studies of traditional CI therapy. ${ }^{4,5}$ This is in contrast to no significant performance improvement on the ARAT. It is possible that the nature of motor practice in Recovery Rapids facilitates improvements in performance speed of the upper limb but not in QOM during grasp, grip, pinch, and gross movements as quantified by the ARAT.

This study contributes substantially to the very small literature on in-home rehabilitation of the upper limb through gaming. Four small-sample prospective studies have demonstrated inconsistent improvements in motor function, ${ }^{15,17-19}$ absent improvement in perceived ability to do real-world tasks ${ }^{18}$ or increased use of the weaker arm for daily activities. ${ }^{17}$ Although adherence to CI therapy transfer package techniques was incomplete, their incorporation into the protocol may explain better carryover of treatment gains to daily activity in the current study relative to prior work.

The study-specific patient advisory group indicated that somatosensation was important (Table 2) so two measures of upper limb somatosensation were used to examine whether motor practice administered through a virtual reality game that lacks a tactile endpoint (eg, handling an object) could improve sensory performance. Neither measure of sensory performance was statistically different at posttest; however, it was notable that the 4 participants with the most severe touch impairment at pretest had improved by one category at posttest. This finding raises the possibility that large doses of upper extremity activity in the chronic phase of stroke can result in improved tactile perception, even though tactile input was not a component of the motor practice. If this finding can be replicated in a larger sample, it will have important implications for sensorimotor rehabilitation. 


\section{Study Limitations}

This pilot study shares many of the same limitations of other early-stage work, including small sample size and no comparison group. Therefore, the improvements found could theoretically result from a learning effect due to familiarization with the outcome measures. Ongoing research will build upon this initial effort by examining a causative relationship between the game-based CI therapy intervention and clinical outcomes through a multisite randomized controlled trial, modifying the delivery of the transfer package using patient-centered approaches to enhance carryover, and performing a well-powered subgroup analysis to determine the influence of somatosensory and cognitive functioning on outcomes. ${ }^{23}$

\section{CONCLUSIONS}

It is feasible to deliver constraint-induced movement therapy to persons with chronic hemiparesis with minimal direct therapist supervision through an in-home video game. Participants' subjective responses suggest the intervention was acceptable. Favorable changes in performance speed and quality of arm use were found in this prospective cohort study, suggesting that randomized comparative-effectiveness trials are warranted.

\section{Patient-Friendly Recap}

- Individuals with hand and arm weakness due to conditions such as stroke often do not have access to the most effective clinic-based rehabilitation methods.

- Computer games enable hand and arm exercises to be done in the home. The authors investigated the potential effectiveness of in-home rehab using a customizable virtual reality video game.

- In this preliminary study the video game was feasible to play in-home, no one was injured, and some study participants experienced improved arm function.

\section{Author Contributions}

Study design: Crawfis, McPherson, Worthen-Chaudhari, Gauthier. Data acquisition or analysis: Borstad, Phillips, Pax Lowes, Siles, Worthen-Chaudhari, Gauthier. Manuscript drafting: Borstad, Gauthier. Critical revision: Crawfis, Phillips, Pax Lowes, Maung, McPherson, Siles, Worthen-Chaudhari.

\section{Conflicts of Interest}

Authors Borstad, Crawfis, Lowes, Maung, McPherson, and Gauthier are shareholders of a company, Games That Move You PBC (Columbus, $\mathrm{OH}$ ), that will complete development and disseminate the video game Recovery Rapids, which is used as the intervention in this study. The other authors have no relevant disclosures.

\section{Funding Sources}

The Patient-Centered Outcomes Research Institute (Washington, DC) awarded a grant (no number assigned) to principal investigator Lynne Gauthier for the study of a low-cost virtual reality gaming platform for neurorehabilitation of hemiparesis. Content is solely the responsibility of the authors and does not necessarily represent the official view of PCORI.

\section{References}

1. Nichols-Larsen DS, Clark PC, Zeringue A, Greenspan A, Blanton S. Factors influencing stroke survivors' quality of life during subacute recovery. Stroke. 2005;36:1480-4. CrossRef

2. Broeks JG, Lankhorst GJ, Rumping K, Prevo AJ. The longterm outcome of arm function after stroke: results of a follow-up study. Disabil Rehabil. 1999;21:357-64.

3. Taub E, Miller NE, Novack TA, et al. Technique to improve chronic motor deficit after stroke. Arch Phys Med Rehabil. 1993;74:347-54.

4. Park SW, Wolf SL, Blanton S, Winstein C, Nichols-Larsen DS. The EXCITE trial: predicting a clinically meaningful motor activity log outcome. Neurorehabil Neural Repair. 2008;22:486-93. CrossRef

5. Taub E, Uswatte G, King DK, Morris D, Crago JE, Chatterjee A. A placebo-controlled trial of constraint-induced movement therapy for upper extremity after stroke. Stroke. 2006;37:1045-9. CrossRef

6. Gauthier LV, Taub E, Perkins C, Ortmann M, Mark VW, Uswatte G. Remodeling the brain: plastic structural brain changes produced by different motor therapies after stroke. Stroke. 2008;39:1520-5. CrossRef

7. Liepert J, Bauder H, Wolfgang HR, Miltner WH, Taub E, Weiller C. Treatment-induced cortical reorganization after stroke in humans. Stroke. 2000;31:1210-6. CrossRef

8. Hakkennes S, Keating JL. Constraint-induced movement therapy following stroke: a systematic review of randomised controlled trials. Aust J Physiother. 2005;51:221-31. $\underline{\text { CrossRef }}$

9. Peurala SH, Kantanen MP, Sjögren T, Paltamaa J, Karhula M, Heinonen A. Effectiveness of constraintinduced movement therapy on activity and participation after stroke: a systematic review and meta-analysis of randomized controlled trials. Clin Rehabil. 2012;26:209-23. CrossRef

10. Wolf SL, Winstein CJ, Miller JP, et al. Retention of upper limb function in stroke survivors who have received constraint-induced movement therapy: the EXCITE randomised trial. Lancet Neurol. 2008;7:33-40. CrossRef

11. Viana R, Teasell R. Barriers to the implementation of constraint-induced movement therapy into practice. Top Stroke Rehabil. 2012;19:104-14. CrossRef

12. Page SJ, Levine P, Sisto S, Bond Q, Johnston MV. Stroke patients' and therapists' opinions of constraint-induced movement therapy. Clin Rehabil. 2002;16:55-60. rossRef 
13. Huber M, Rabin B, Docan C, Burdea GC, AbdelBaky M, Golomb MR. Feasibility of modified remotely monitored in-home gaming technology for improving hand function in adolescents with cerebral palsy. IEEE Trans Inf Technol Biomed. 2010;14:526-34. CrossRef

14. Sandlund M, Dock K, Häger CK, Waterworth EL. Motion interactive video games in home training for children with cerebral palsy: parents' perceptions. Disabil Rehabil. 2012;34:925-33. CrossRef

15. Lin J, Kelleher CL, Engsberg JR. Developing home-based virtual reality therapy interventions. Games Health $J$. 2013;2:34-8. CrossRef

16. Standen PJ, Threapleton K, Connell L, et al. Patients' use of a home-based virtual reality system to provide rehabilitation of the upper limb following stroke. Phys Ther. 2015;95:350-9. CrossRef

17. Nijenhuis SM, Prange GB, Amirabdollahian F, et al. Feasibility study into self-administered training at home using an arm and hand device with motivational gaming environment in chronic stroke. $J$ Neuroeng Rehabil. 2015;12:89. CrossRef

18. Slijper A, Svensson KE, Backlund $\mathrm{P}$, Engström $\mathrm{H}$, Sunnerhagen KS. Computer game-based upper extremity training in the home environment in stroke persons: a single subject design. J Neuroeng Rehabil. 2014;11:35. CrossRef

19. Donoso Brown EV, McCoy SW, Fechko AS, Price R, Gilbertson T, Moritz CT. Preliminary investigation of an electromyography-controlled video game as a home program for persons in the chronic phase of stroke recovery. Arch Phys Med Rehabil. 2014;95:1461-9. CrossRef

20. Wingham J, Adie K, Turner D, Schofield C, Pritchard C. Participant and caregiver experience of the Nintendo Wii SportsTM after stroke: qualitative study of the trial of WiiTM in stroke (TWIST). Clin Rehabil. 2015;29:295-305. CrossRef

21. Taub E, Uswatte G, Mark VW, et al. Method for enhancing real-world use of a more affected arm in chronic stroke: transfer package of constraint-induced movement therapy. Stroke. 2013;44:1383-8. CrossRef

22. Morris DM, Taub E, Mark VW. Constraint-induced movement therapy: characterizing the intervention protocol. Eura Medicophys. 2006;42:257-68.

23. Gauthier LV, Kane C, Borstad A, et al. Video Game Rehabilitation for Outpatient Stroke (VIGoROUS): protocol for a multi-center comparative effectiveness trial of inhome gamified constraint-induced movement therapy for rehabilitation of chronic upper extremity hemiparesis. $B M C$ Neurol. 2017;17(1):109. CrossRef

24. Wolf SL, Winstein CJ, Miller JP, et al. Effect of constraintinduced movement therapy on upper extremity function 3 to 9 months after stroke: the EXCITE randomized clinical trial. JAMA. 2006;296:2095-104. CrossRef

25. Maung D, Crawfis R, Gauthier LV, et al. Games for therapy: defining a grammar and implementation for the recognition of therapeutic gestures. Proceedings of the 8th International Conference on the Foundations of Digital Games (FDG 2013). Santa Cruz, CA: Society for the Advancement of the Science of Digital Games, 2013, pp. 314-21.

26. Maung D, Crawfis R, Gauthier LV, et al. Development of Recovery Rapids - a game for cost effective stroke therapy. Proceedings of the 9th International Conference on the Foundations of Digital Games. Santa Cruz, CA: Society for the Advancement of the Science of Digital Games, 2014.
27. Gauthier LV, Borstad A, Luong E, et al. Upper extremity recovery following gamified constraint-induced movement therapy: a case study in dense amnestic TBI. Brain Injury Professional. 2016;12(4).

28. Kroenke K, Spitzer RL, Williams JB. The PHQ-9: validity of a brief depression severity measure. J Gen Intern Med. 2001;16:606-13. CrossRef

29. Nasreddine ZS, Phillips NA, Bédirian V, et al. The Montreal Cognitive Assessment, MoCA: a brief screening tool for mild cognitive impairment. J Am Geriatr Soc. 2005;53:695-9. CrossRef

30. Kroenke K, Spitzer RL. The PHQ-9: a new depression diagnostic and severity measure. Psychiatr Ann. 2002;32:509-15. CrossRef

31. Devine EB, Alfonso-Cristancho R, Devlin A, et al. A model for incorporating patient and stakeholder voices in a learning health care network: Washington State's Comparative Effectiveness Research Translation Network. $J$ Clin Epidemiol. 2013;66(8 Suppl):S122-9. CrossRef

32. Hodics TM, Nakatsuka K, Upreti B, Alex A, Smith PS, Pezzullo JC. Wolf Motor Function Test for characterizing moderate to severe hemiparesis in stroke patients. Arch Phys Med Rehabil. 2012;93:1963-7. CrossRef

33. Taub E, Uswatte G, Pidikiti R. Constraint-Induced Movement Therapy: a new family of techniques with broad application. J Rehabil Res Dev. 1999;36:237-51.

34. Lyle RC. A performance test for assessment of upper limb function in physical rehabilitation treatment and research. Int J Rehabil Res. 1981;4:483-92. CrossRef

35. Platz T, Pinkowski C, van Wijck F, Kim IH, di Bella P, Johnson G. Reliability and validity of arm function assessment with standardized guidelines for the Fugl-Meyer Test, Action Research Arm Test and Box and Block Test: a multicentre study. Clin Rehabil. 2005;19:404-11. CrossRef

36. Van der Lee JH, De Groot V, Beckerman H, Wagenaar RC, Lankhorst GJ, Bouter LM. The intra-and interrater reliability of the action research arm test: a practical test of upper extremity function in patients with stroke. Arch Phys Med Rehabil. 2001;82:14-9. CrossRef

37. National Institutes of Health. Attachment 15. Brief Kinesthesia Test. http://www.reginfo.gov/public/do/ DownloadDocument?objectID=25142401. Accessed October 10, 2012.

38. Dunn W, Griffith JW, Morrison MT, et al. Somatosensation assessment using the NIH Toolbox. Neurology. 2013;80(11 Suppl 3):S41-4. CrossRef

39. Borstad A, Nichols-Larsen DS. The Brief Kinesthesia test is feasible and sensitive: a study in stroke. Braz J Phys Ther. 2016;20:81-6. CrossRef

40. Hunter JM, Mackin EJ, Callahan AD. Rehabilitation of the Hand: Surgery and Therapy, Fourth Edition. Boca Raton, FL: CRC Press, 1995.

41. Novak CB, Mackinnon SE, Williams JI, Kelly L. Establishment of reliability in the evaluation of hand sensibility. Plast Reconstr Surg. 1993;92:311-22. CrossRef

42. Halar EM, Hammond MC, LaCava EC, Camann C, Ward J. Sensory perception threshold measurement: an evaluation of semiobjective testing devices. Arch Phys Med Rehabil. 1987;68:499-507.

43. Rolke R, Magerl W, Campbell KA, et al. Quantitative sensory testing: a comprehensive protocol for clinical trials. Eur $J$ Pain. 2006;10:77-88. $\underline{\text { CrossRef }}$ 
44. Cohen J. A power primer. Psychol Bull. 1992;112:155-9. CrossRef

45. Portney LG, Watkins MP. Foundations of Clinical Research: Applications to Practice, Third Edition. Upper Saddle River, NJ: Prentice Hall, 2009.

46. Cohen J, Cohen P, West SG, Aiken LS. Applied Multiple Regression/Correlation Analysis for the Behavioral Sciences, Third Edition. Mahwah, NJ: Lawrence Erlbaum Associates, 2003.

47. Lang CE, Strube MJ, Bland MD, et al. Dose response of task-specific upper limb training in people at least 6 months poststroke: a phase II, single-blind, randomized, controlled trial. Ann Neurol. 2016;80:342-54. CrossRef

48. van der Lee JH, Beckerman H, Knol DL, de Vet HC, Bouter LM. Clinimetric properties of the motor activity log for the assessment of arm use in hemiparetic patients. Stroke. 2004;35:1410-4. CrossRef

49. Combs SA, Finley MA, Henss M, Himmler S, Lapota K, Stillwell D. Effects of a repetitive gaming intervention on upper extremity impairments and function in persons with chronic stroke: a preliminary study. Disabil Rehabil. 2012;34:1291-8. $\underline{\text { CrossRef }}$
50. Kleim JA, Barbay S, Cooper NR, et al. Motor learning-dependent synaptogenesis is localized to functionally reorganized motor cortex. Neurobiol Learn Mem. 2002;77:63-77. CrossRef

51. Brogårdh $\mathrm{C}$, Vestling $\mathrm{M}$, Sjölund $\mathrm{BH}$. Shortened constraintinduced movement therapy in subacute stroke - no effect of using a restraint: a randomized controlled study with independent observers. J Rehabil Med. 2009;41:231-6.

52. Gauthier L. CI therapy transfer package. https://www. youtube.com/watch? $\mathrm{v}=$ Dlwk5uV3gKY\&t=23s. Accessed December 7, 2017.

53. Gauthier L. The research behind CI therapy. https://www. youtube.com/watch? $\mathrm{v}=7 \mathrm{VqfGTLIp} 88 \& \mathrm{t}=187 \mathrm{~s}$. Accessed December 7, 2017.

(C) 2018 Aurora Health Care, Inc. 